\title{
Dieta cetogénica: mecanismos en el control de la epilepsia refractaria
}

\author{
Ketogenic diet: refractory epilepsy control mechanisms
}

\author{
Rodolfo Sanzana-Cuche' , Tomás Labbé ${ }^{1}$
}

\section{Resumen}

Introducción: Existe un porcentaje relevante de pacientes con epilepsia que son refractarios al tratamiento con fármacos anticonvulsivantes. Este cuadro, se denomina epilepsia refractaria (ER), en el cual, la dieta cetogénica (DC), ha probado ser una alternativa efectiva. Existen diferentes versiones de la DC, y ciertos componentes de esta dieta han mostrado ser más efectivos que otros. Por otra parte, diferentes autores han propuesto hipótesis, de cómo, la DC podría actuar a nivel molecular y celular, para controlar estas crisis epilépticas. Los objetivos de esta revisión, son, explicar la DC, y analizar la evidencia existente, en relación con los mecanismos, a través de los cuales, la DC ejerce un control de las crisis convulsivas. Método y resultados: Se realizó una búsqueda avanzada en PubMed, considerando los términos «refractory», «epilepsy», «ketogenic»y «diet», incluidos en el título y el resumen. La búsqueda, dio como resultado, 288 artículos publicados en los últimos 10 años. De estos artículos, se seleccionaron 134 potencialmente relevantes, posterior a la lectura de sus títulos. Se realizó la lectura del resumen para los 134 artículos, de los cuales, se seleccionaron 17, que fueron, a juicio de los autores, los que incluían la información pertinente para esta revisión. La búsqueda se centró en artículos originales y revisiones disponibles en inglés, con énfasis, en los artículos apropiados para comprender los conceptos generales de la DC y los mecanismos de acción, que podrían representar un rol relevante al momento de controlar la ER. Conclusiones: La DC ha probado ser efectiva en el tratamiento de la ER, sin embargo, hay pacientes que no la toleran adecuadamente. Existen ciertos compuestos asociados la DC que han probado ser más efectivos que otro como es el caso de los triglicéridos de cadena mediana (TCM). Finalmente, se han investigado numerosas hipótesis en relación con el mecanismo de acción de la DC, donde destacan las hipótesis de los cuerpos cetónicos, la adenosina y la del cortocircuito GABA.

Palabras clave: dieta cetogénica; epilepsia; epilepsia refractaria; cuerpos cetónicos; convulsiones; anticonvulsivantes.

\begin{abstract}
Introduction: A percentage of epilepsy patients are refractory to treatment with anticonvulsant drugs. An effective alternative for treating this condition, known as refractory epilepsy (RE), is the ketogenic diet (KD). There are different variations of the KD, and certain components of this diet have been shown more effective than others. Various authors have proposed hypotheses for how the KD acts on the molecular and cellular levels in controlling seizures. The objectives of this review are to explain the KD and analyze existing evidence regarding the mechanisms through which the KD exercises control over seizures. Methods and results: PubMed was extensively searched using the terms «refractory», «epilepsy», "ketogenic», and «diet» included in the title and abstract. The search returned 288 published articles within the last ten years. Of these 288 manuscripts, 134 were selected as potentially relevant based on the title. After reading the abstract of each potentially relevant paper, 17 were finally included in the review. These manuscripts, per the judgement of the authors of this study, included information pertinent to the review. The search focused on original articles and reviews available in English, with an emphasis on texts appropriate for understanding the general concepts of the KD and the mechanisms of action that could play a relevant role in controlling RE. Conclusions: The KD has been proven effective in treating RE. However, some patients do not adequately tolerate this diet. There are certain compounds associated with the KD that are more effective than others, as is the case with medium-chain triglycerides. Finally, numerous hypotheses exist regarding the mechanisms of action for the KD. Notable among these hypotheses are ketone bodies, adenosine, and the GABA shunt.
\end{abstract}

Keywords: ketogenic diet; epilepsy; refractory epilepsy; ketone bodies; seizures; anticonvulsants.

Fecha de envío: 11 de agosto de 2019 - Fecha de aceptación: 16 de marzo de 2020

(1) Escuela de Medicina. Facultad de Medicina y Ciencia, Universidad San Sebastián, Santiago, Chile.

Autor de correspondencia: tplabbe@uc.cl 


\section{Introducción}

La epilepsia, es una enfermedad del cerebro, que se define por cualquiera de las siguientes tres circunstancias: a) al menos, dos crisis epilépticas no provocadas (o reflejas), separadas por más de 24 horas, b) una crisis epiléptica no provocada (o refleja), asociada a un riesgo (de al menos un $60 \%$ de probabilidad de recurrencia en los próximos 10 años) de presentar nuevas crisis, similar al derivado tras tener dos crisis no provocadas y por último, c) el diagnóstico de un síndrome epiléptico (Fisher et al., 2014). Estas crisis, son episodios breves de movimientos involuntarios, que pueden afectar, a una parte del cuerpo (crisis parciales) o a su totalidad (crisis generalizadas) y a veces, se acompañan de pérdida de la consciencia y del control de los esfínteres. Se estima, que más de 50 millones de personas a nivel mundial, padecen de epilepsia, y que aproximadamente el $80 \%$ vive en países de bajos y medianos ingresos (Megiddo et al., 2016).

La Comisión de Clasificación y Terminología de la Liga Internacional Contra la Epilepsia, ha actualizado la clasificación del 2010 (Scheffer et al., 2017), y ha establecido un marco, el cual se define en relación con tres niveles diagnósticos, asumiendo que el clínico, ya ha realizado un diagnóstico definitivo de una crisis epiléptica. El nivel 1, implica determinar el tipo de crisis epiléptica, la cual puede ser de inicio focal, inicio generalizado o de inicio desconocido, esta última, se aplica cuando el clínico reconoce que hay una crisis propiamente tal, pero no tiene evidencia suficiente que sustente una clasificación determinada. El nivel 2, corresponde a la identificación del tipo de epilepsia, la cual puede ser focal, generalizada, una combinación de focal y generalizada o desconocida, todo lo anterior, asumiendo que el paciente tiene un diagnóstico de epilepsia acorde con la definición del 2014 (Fisher et al., 2014). El nivel 3, implica el diagnóstico de un síndrome epiléptico, el cual, se define como un cuadro de características clínicas, signos y síntomas, que, en conjunto, definen un desorden clínico convulsivo reconocible y distintivo. Por último, es fundamental reconocer la etiología de la epilepsia. Estas, pueden ser diversas, y por lo general, la primera que se descarta, con un examen imagenológico de resonancia magnética, corresponde a la etiología estructural. El resto, son las genéticas, inmunes, infecciosas, metabólicas y las desconocidas (Scheffer et al., 2017). A pesar de generar condiciones inhabilitantes, esta patología puede ser controlada en la mayoría de los pacientes con uno o más fármacos anticonvulsivantes (D’Andrea Meira et al., 2019). No obstante, lo anterior, un $30 \%$ de los pacientes son categorizados con epilepsia refractaria (ER). Esta última, se define como un control insuficiente de las crisis, a pesar de haberse prescrito una terapia adecuada, con una combinación de 2 o más fármacos anticonvulsivantes apropiadamente seleccionados (Ułamek-Kozioł et al., 2016; D’Andrea Meira et al., 2019). Se ha descrito, que una de las principales etiologías asociadas con la ER, es la genética. En relación con esta etiología, existen mutaciones que afectan diferentes estructuras y/o vías dentro del sistema nervioso, tales como: a) alteraciones genéticas que comprometen canales y la transmisión sináptica, como los canales dependiente de sodio y los receptores AMPA, b) alteraciones genéticas que alteran la vía mTOR, involucrada en procesos tales como, crecimiento neuronal, migración y proliferación y c) microdeleciones y alteraciones cromosomales relacionadas con la epilepsia (Helbig et al., 2008; Stevelink et al., 2018). El único tratamiento, que es parcial o totalmente curativo en la ER, es la cirugía, sin embargo, esta es subutilizada (De Flon et al., 2010), y en general menos de la mitad de pacientes con ER son remitidos para ser evaluados como eventuales candidatos a una cirugía (Uijl et al., 2012). Dentro de las alternativas de tratamiento a la ER, se incluyen, la estimulación del nervio vago, fármacos anticonvulsivantes de nueva generación, estimulación cortical y la dieta cetogénica (Vaccarezza \& Silva, 2015). Esta última, consiste en un tratamiento no farmacológico incluido en diversas guías de práctica clínica (Armeno et al., 2014; Minsal, 2014). La DC, presenta indicaciones en pacientes con ER y patologías metabólicas como: el síndrome de deficiencia del transportador de glucosa 1 (GLUT-1), la deficiencia de la piruvato deshidrogenasa y la patología mitocondrial. La DC es alta en grasas, considerando un aporte total del 71-90\% del total de ingesta alimentaria durante el día. A lo anterior, se asocia una restricción en la ingesta de carbohidratos, considerando un aporte máximo, del orden de 5-19\%, incluyendo un aporte adecuado de proteínas, de acuerdo con los requerimientos nutricionales del paciente (Van der Louw et al., 2016; Minsal, 2019). Los objetivos de esta revisión, son, explicar la DC, y analizar la evidencia existente, en relación con los mecanismos, a través de los cuales, la DC ejerce un control de las crisis convulsivas.

\section{Dieta cetogénica}

\section{Historia y conceptos generales}

Si bien la DC es una de las terapias no farmacológicas más difundidas para la ER, esta comenzó a ser utilizada sistemáticamente desde la década de 1920. Existen registros que el ayuno permitía el control de las crisis convulsivas incluso desde la era Hipocrática (460 AC. - 370 AC.) (Sampaio, 2016). En 1921, el Dr. Wilder, en la Clínica Mayo en Minnesota, Estados Unidos, impulsó su uso contemporáneo. El objetivo inicial fue identificar un tratamiento alternativo a los pocos fármacos anticonvulsivantes disponibles en la época (Wilder, 1921; Kossoff \& Hartman, 2012). En el 1938, con la inclusión de la fenitoína como fármaco anticonvulsivante, disminuye el interés de la comunidad científica y clínica en la DC, reapareciendo el interés por su efectividad en la década de 1990. En este último período, se robustece la evidencia de la DC como una herramienta efectiva para controlar las crisis que son resistentes a las drogas anticonvulsivantes. 


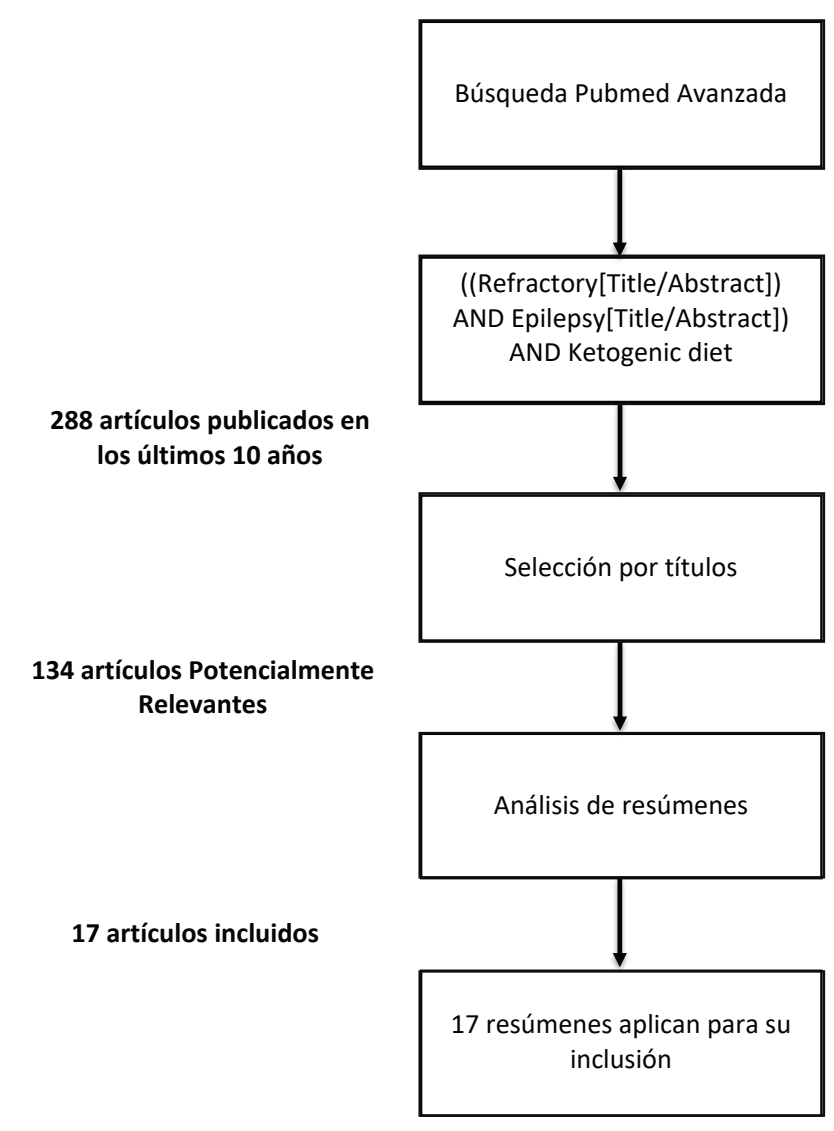

Figura 1: Diagrama de flujo de la estrategia de búsqueda.

\section{¿Qué tipo de grasa consumir en la DC?}

La DC de triglicéridos de cadena media (TCM), ha probado ser, uno de los enfoques terapéuticos más efectivos en niños con ER (Kossoff et al., 2009). El esquema, sobre la base de TCM, fue desarrollado para elaborar una propuesta dietética más tolerable, permitiendo, además, una dieta con una mayor proporción de carbohidratos y proteínas. Lo anterior, en función de que los TCM producen más cetonas por kilocaloría de energía que los triglicéridos de cadena larga usados en la forma clásica de DC. De esta manera, esta terapia requiere menor ingesta de grasas para generar niveles similares de cetonas respecto de la DC clásica. Por otro lado, es conveniente destacar que existen numerosos efectos adversos asociados a la DC de TCM, tales como, diarrea, vómitos y dolor abdominal, es por esto que la DC de TCM ha sufrido modificaciones para hacerla más tolerable, modificando el porcentaje de TCM, llegando a 30 $\%$ de TCM y $30 \%$ de triglicéridos de cadena larga. De esta forma, se busca incrementar progresivamente el componente de interés hasta los máximos niveles tolerados (Sampaio, 2016).

En relación con la eficacia que se ha visto en la DC de TCM y a los numerosos efectos adversos del ácido valproico (ácido graso de cadena corta y uno de los principales anticonvulsivantes usados en un amplio espectro de crisis epilépticas), como teratogenicidad y hepatotoxicidad, se ha estado investigando en nuevas alternativas terapéuticas. Se ha observado, que el ácido decanoico, un TCM lineal de 10 carbonos, tuvo un fuerte control de las crisis convulsivas, en relación con el ácido valproico (Chang et al., 2013). Por otro lado, el uso de ácido octanoico de 8 carbonos, no aumentó significativamente el control de las crisis. De esta manera, existen diferentes esquemas dietéticos en estudio que pretenden mejorar la seguridad que se obtiene con los esquemas terapéuticos actuales. En virtud de la eficacia de las grasas anteriormente mencionadas, es que se han propuesto diversas hipótesis de cómo las grasas podrían controlar las convulsiones en la epilepsia.

\section{Hipótesis funcionales sobre el efecto de la dieta cetogénica} Existen diferentes hipótesis que se han postulado para explicar el mecanismo anticonvulsivante de la DC, incluyendo las hipótesis del $\mathrm{pH}$, metabólicas, de cortocircuito GABA, de cetonas, de adenosina, de restricción calórica, de los lípidos cerebrales y la de la noradrenalina (Stafstrom \& Rho, 2004; Nylen et al., 2009). De las diferentes hipótesis mencionadas anteriormente, nos concentramos en las hipótesis de cetonas, adenosina y de cortocircuito GABA, que exhiben un rol predominante en la literatura consultada.

\section{Cuerpos cetónicos}

Una de las hipótesis del control de las convulsiones por parte de la DC, es la elevada concentración plasmática de cuerpos cetónicos (acetona, acetoacetato y $\beta$-hidroxibutirato) en sangre. Sin embargo, no se han podido demostrar los mecanismos exactos, de cómo, los cuerpos cetónicos, modulan la actividad eléctrica. Existe evidencia (Masino \& Rho, 2012), asociada a su acción a nivel mitocondrial, la cual, ha probado ser, por sobre todo efectiva en pacientes jóvenes, probablemente por la expresión de transportadores de monocarboxilato, quienes importan los cuerpos cetónicos a través de la barrera hematoencefálica desde la circulación sistémica (Morris, 2005).

\section{Adenosina}

Se conoce, que la adenosina actúa sobre los receptores A1 (A1R) y se considera, que posee un efecto marcadamente anticonvulsivante. Es fundamental entonces, considerar a la adenosina endógena, como un mecanismo de control de convulsiones (Etherington \& Frenguelli, 2004). En concordancia con lo anterior, un aumento en el nivel de adenosina cinasa o una disminución en la expresión de $A 1 R$, se relacionan con convulsiones electroencefalográficas espontáneas a nivel intrahipocampal (Li et al., 2008).

\section{Cortocircuito GABA}

Es bien conocida, la vía indirecta asociada al ciclo de Krebs que sigue GABA para su síntesis. En resumen, desde alfa-cetoglutarato, 
se forma glutamato (Neurotransmisor excitatorio), lo que luego da origen a GABA, succinil semialdehído y finalmente succinato (Lodish et al., 2000). Esta propuesta, apunta principalmente, a que en la DC existe un aumento de acetil coenzima A derivada del metabolismo de las grasas, por lo que existiría, un aumento en la producción de alfa-cetoglutarato, lo que conlleva un aumento en la síntesis de glutamato, pero por sobre todo de GABA, que gracias a su efecto inhibitorio, ejercería un efecto regulador en las convulsiones (Nylen et al., 2009). La tabla 1 resume los mecanismos involucrados en las principales hipótesis.

Tabla 1: Moléculas y mecanismos de acción de las diferentes hipótesis de la dieta cetogénica.

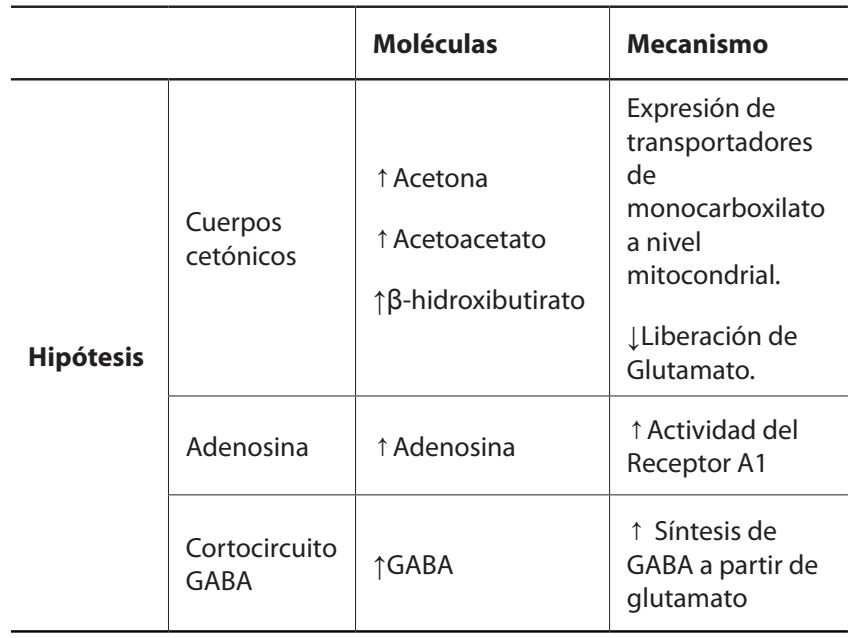

\section{Discusión y Conclusiones}

La DC, ha probado ser una herramienta terapéutica efectiva para tratar de forma no farmacológica la ER. Hasta la fecha, no están bien establecidos los mecanismos exactos, a través de los cuales, las DC ejerce sus efectos anticonvulsivantes, sin embargo, varias hipótesis han sido propuestas. De las diferentes hipótesis analizadas, la hipótesis de los cuerpos cetónicos, plantea un aumento en la expresión de transportadores de monocarboxilato a nivel mitocondrial y un aumento en la liberación de Glutamato. Sin embargo, la concentración plasmática de cetonas tiene una pobre correlación con el control de las convulsiones en la mayoría de estudios clínicos y de animales (Kawamura et al., 2014). Últimamente, se ha demostrado, que la exposición aguda a $\beta$-hidroxibutirato, no es tan efectiva como una exposición más crónica (Kim et al., 2015), lo que sugeriría, que se requiere una adaptación a los mecanismos de acción de los cuerpos cetónicos. Otros autores (Juge et al., 2010), han demostrado que los cuerpos cetónicos, acetoacetato e $\beta$-hidroxibutirato, disminuyeron la liberación de glutamato, compitiendo directamente con cloruro, por la activación alostérica de vesículas transportadoras de glutamato.
Por otra parte, la hipótesis en relación con la adenosina, sugiere un aumento en el nivel de adenosina cinasa o una disminución en la expresión de A1R (Li et al., 2008), sin embargo, ciertos autores, hipotetizan que la DC aumenta los niveles de adenosina a nivel cerebral, lo cual, fue evidenciado en un estudio realizado en ratones transgénicos Adk-Tg (con niveles cerebrales aumentados de adenosina cinasa), A1R +/- y A1R-/-, donde la DC, logró disminuir significativamente la cantidad y el tiempo de convulsiones en ratones Adk-Tg, lo propio se logró en los ratones A1R+/-, pero en menor medida que en los ratones Adk-Tg. Finalmente, apoyando esta hipótesis, la DC no logró generar cambios en los ratones A1R-/(Masino et al., 2011).

Por último, en relación con la hipótesis de cortocircuito GABA asociada al aumento de acetil coenzima A derivada del metabolismo de las grasas (Nylen et al., 2009), se propone además, que existen cambios en el metabolismo del glutamato, en este sentido, se describe que este neurotransmisor, es removido del espacio sináptico por los astrocitos, los cuales convierten al glutamato en glutamina por vía de la acción de la enzima glial glutamina sintasa. Glutamina, es entonces exportada a neuronas, donde es hidrolizada a glutamato, el cual podría ser convertido a GABA o transaminado a aspartato en una reacción que requiere oxalacetato. Debido, a que la DC induce cambios metabólicos que requieren oxalacetato para reaccionar con acetil coenzima A para ser incorporada al ciclo de Krebs, la producción de aspartato desde glutamato está reducida. Esto podría significar, un mayor flujo a través de la enzima sintetizadora de GABA, glutamato decarboxilasa, para aumentar la síntesis de GABA (Rogawski et al., 2016).

La comprensión, precisa y acabada de los mecanismos involucrados en las diferentes hipótesis, conducirá a una adecuada aplicación clínica de la DC y a un creciente número de investigaciones.

\section{Referencias}

Armeno M, Caraballo RH, Vaccarezza Ferrari M, Alberti MJ, Ríos V, Galicchio, S, De Grandis, E. S, Mestre, G, Escobal N, Matarrese P, Viollaz R, Agostinho A, Díez C, Cresta A, Cabrera A, Blanco V, Ferrero $\mathrm{H}$, Gambarini V, Sosa P, et al. (2014). Consenso nacional sobre dieta cetogénica. Revista de Neurología, 59, 213.

Chang P, Terbach N, Plant N, Chen PE, Walker MC \& Williams RSB. (2013). Seizure control by ketogenic diet-associated medium chain fatty acids. Neuropharmacology, 69, 105-114.

D’Andrea Meira I, Romão TT, Pires do Prado HJ, Krüger LT, Pires MEP \& Da Conceição PO. (2019). Ketogenic Diet and Epilepsy: What We Know So Far. Frontiers in Neuroscience 13, 5. 
De Flon P, Kumlien E, Reuterwall C. \& Mattsson P. (2010). Empirical evidence of underutilization of referrals for epilepsy surgery evaluation. European Journal of Neurology: The Official Journal of the European Federation of Neurological Societies 17, 619-625.

Etherington LAV \& Frenguelli BG. (2004). Endogenous adenosine modulates epileptiform activity in rat hippocampus in a receptor subtype-dependent manner. The European Journal of Neuroscience 19, 2539-2550.

Fisher RS, Acevedo C, Arzimanoglou A, Bogacz A, Cross JH, Elger CE, Engel J, Jr Forsgren L, French JA, Glynn M, Hesdorffer DC, Lee BI, Mathern GW, Moshé SL, Perucca E, Scheffer IE, Tomson T, Watanabe M \& Wiebe S. (2014). ILAE official report: a practical clinical definition of epilepsy. Epilepsia 55, 475-482.

Helbig I, Scheffer IE, Mulley JC \& Berkovic SF. (2008). Navigating the channels and beyond: unravelling the genetics of the epilepsies. Lancet Neurology 7, 231-245.

Juge N, Gray JA, Omote H, Miyaji T, Inoue T, Hara C, Uneyama H, Edwards RH, Nicoll RA. \& Moriyama Y. (2010). Metabolic control of vesicular glutamate transport and release. Neuron 68, 99-112.

Kawamura M Jr, Ruskin DN, Geiger JD, Boison D \& Masino SA. (2014). Ketogenic diet sensitizes glucose control of hippocampal excitability. Journal of Lipid Research 55, 2254-2260.

Kim DY, Simeone KA, Simeone TA, Pandya JD Wilke JC, Ahn Y, Geddes JW, Sullivan PG \& Rho JM. (2015). Ketone bodies mediate antiseizure effects through mitochondrial permeability transition. Annals of Neurology 78, 77-87.

Kossoff EH \& Hartman AL. (2012). Ketogenic diets: new advances for metabolism-based therapies. Current Opinion in Neurology 25, 173-178.

Kossoff EH, Zupec-Kania BA. \& Rho JM. (2009). Ketogenic diets: an update for child neurologists. Journal of Child Neurology 24, 979-988.

Li T, Ren G, Lusardi T, Wilz A, Lan JQ, Iwasato T, Itohara S, Simon RP \& Boison D. (2008). Adenosine kinase is a target for the prediction and prevention of epileptogenesis in mice. The Journal of Clinical Investigation 118, 571-582.

Lodish H, Berk A, Lawrence Zipursky S, Matsudaira P, Baltimore D \& Darnell J. (2000). Molecular Cell Biology. W. H. Freeman.

Masino SA, Li T, Theofilas P, Sandau US, Ruskin DN, Fredholm, BB, Geiger JD, Aronica E \& Boison D. (2011). A ketogenic diet suppresses seizures in mice through adenosine $A_{1}$ receptors. The Journal of Clinical Investigation 121, 2679-2683.
Masino SA \& Rho JM. (2012). Mechanisms of Ketogenic Diet Action. In J. L. Noebels, M. Avoli, M. A. Rogawski, R. W. Olsen, \& A. V. Delgado-Escueta (Eds.), Jasper's Basic Mechanisms of the Epilepsies. National Center for Biotechnology Information (US).

Megiddo I, Colson A, Chisholm D, Dua T, Nandi A \& Laxminarayan R. (2016). Health and economic benefits of public financing of epilepsy treatment in India: An agent-based simulation model. Epilepsia 57, 464-474.

Ministerio de Salud. (2014). Guía clínica AUGE epilepsia adultos.

Ministerio de Salud. (2019). Protocolo técnico para el uso de dieta cetogénica en personas con epilepsia refractaria.

Morris AAM. (2005). Cerebral ketone body metabolism. Journal of Inherited Metabolic Disease, 28, 109-121.

Nylen K, Likhodii S \& Burnham WM. (2009). The ketogenic diet: proposed mechanisms of action. Neurotherapeutics: The Journal of the American Society for Experimental NeuroTherapeutics 6, 402-405.

Rogawski MA, Löscher W \& Rho JM. (2016). Mechanisms of Action of Antiseizure Drugs and the Ketogenic Diet. Cold Spring Harbor Perspectives in Medicine, $\mathbf{6}$.

Sampaio LP de B. (2016). Ketogenic diet for epilepsy treatment. Arquivos de Neuro-Psiquiatria 74, 842-848.

Scheffer IE, Berkovic S, Capovilla G, Connolly MB, French J, Guilhoto L, Hirsch E, Jain S, Mathern GW, Moshé SL, Nordli DR, Perucca E, Tomson T, Wiebe S, Zhang Y-H \& Zuberi S M. (2017). ILAE classification of the epilepsies: Position paper of the ILAE Commission for Classification and Terminology. Epilepsia 58, 512-521.

Stevelink R, Sanders MW, Tuinman MP, Brilstra EH, Koeleman BP, Jansen FE. \& Braun KP. (2018). Epilepsy surgery for patients with genetic refractory epilepsy: a systematic review. Epileptic Disorders: International Epilepsy Journal with Videotape 20, 99-115.

Uijl SG, Leijten, FSS, Moons KGM, Veltman EPH M, Ferrier $\mathrm{CH}$. \& van Donselaar CA. (2012). Epilepsy surgery can help many more adult patients with intractable seizures. Epilepsy Research $101,210-216$.

Ułamek-Kozioł M, Pluta R, Bogucka-Kocka A. \& Czuczwar SJ. (2016). To treat or not to treat drug-refractory epilepsy by the ketogenic diet? That is the question. Annals of Agricultural and Environmental Medicine: AAEM 23, 533-536. 
Vaccarezza MM. \& Silva WH. (2015). Dietary therapy is not the best option for refractory nonsurgical epilepsy. Epilepsia 56, 1330-1334.

Van der Louw E, Van den Hurk D, Neal E, Leiendecker B, Fitzsimmon G, Dority L, Thompson L, Marchió M, Dudzińska M, Dressler A, Klepper J, Auvin S \& Cross JH. (2016). Ketogenic diet guidelines for infants with refractory epilepsy. European Journal of Paediatric Neurology: EJPN: Official Journal of the European Paediatric Neurology Society 20, 798-809.

Wilder RM. (1921). The effects of ketonemia on the course of epilepsy. Mayo Clin Proc 2, 307-308. 